Klagenfurter Geographische Schriften 29 (2013) 11-14

\title{
Einführung der Herausgeber
}

\author{
Béla Filep ${ }^{1}$, Monika M. Váradi ${ }^{2}$, Martin Walter ${ }^{3}$, Rudolf Wastl ${ }^{4}$ \\ ${ }^{\text {I}}$ Geographisches Institut, Universität Bern (bfilep@giub.unibe.ch), ${ }^{2}$ Institut für Regionalforschung, Ungarische \\ AkademiederWissenschaften (varadim@mta-rkk-tko.hu), ${ }^{3}$ Bayerisches Landwirtschaftliches Wochenblatt \\ (wbwien@aon.at), ${ }^{4}$ Institut für Geographie und Regionalforschung, Alpen-Adria Universität Klagenfurt \\ (rudolfwastl@gmx.at)
}

Nie ist es einfach, ein vielschichtiges akademisches Lebenswerk in prägnante Formeln und griffige Titel zu bringen. Dennoch gab es große Einigkeit in der Gruppe der Herausgeber dieser Festschrift zum persönlichen Jubiläum von Univ.-Prof. Doris Wastl-Walter, dass der Ansatz des Überwindens von Grenzen - in manchen Momenten geradezu eine Abscheu vor engstirnigem Denken - ein lebenslanges Lebensprinzip war und ist. Das Motiv von „Grenzüberschreitenden Nachbarschaften" erschien uns daher als die entsprechende Themenstellung.

Und der zweite zentrale Zugang wurde von der Jubilarin selbst immer wieder angesprochen: Nicht persönliche Würdigungen und Ehrungen sind ihr wichtig, sondern Publikationsmöglichkeiten für junge Kolleginnen und Kollegen und erfolgversprechende Studierende.

Die Beiträge zu dieser Festschrift konzentrieren sich deshalb auf die Regionen Mittel- und Südosteuropa, da Doris Wastl-Walter sich seit vielen Jahren mit der Thematik von Grenzen und deren Überwindung in diesem Raum befasst. Bereits zur Zeit des Eisernen Vorhangs hat sie an gemeinsamen Forschungsprojekten mit Kolleginnen und Kollegen aus Ungarn mitgewirkt. Seit bald 15 Jahren initiiert sie selbst zahlreiche Projekte der Europäischen Union und des Schweizerischen Nationalfonds.

Seit dem Fall des Eisernen Vorhangs haben sich tiefgreifende Veränderungen in den Grenzräumen Mittel- und Südosteuropas ergeben. Im Mittelpunkt der Erforschung dieser Vorgänge steht nun, wie die betroffenen Menschen den Abbau von Grenzen (mancherorts auch den Aufbau neuer Grenzen), die neuen Gegebenheiten wahrnehmen, denken, erleben, und wie sie in diesen neuen Strukturen agieren.

Diese Forschungsreihe war Ausgangspunkt für eine Weiterentwicklung theoretischer Konzepte und Methoden zu Grenzforschung und Migration, die an weltweiten Beispielen angewendet wurden. Die entsprechenden Publikationen dazu liegen in der von Doris Wastl-Walter herausgegebenen Ashgate-Reihe „Border Regions Series“ und im international anerkannten Basiswerk „Ashgate Research Companion to Border Studies“"vor.

Die Autorinnen und Autoren, die für die vorliegende Festschrift gewonnen werden konnten, haben in verschiedenen Projekten mit Doris Wastl-Walter zusammengearbeitet und publiziert. Sie stammen aus acht Ländern und unterschiedlichen Sprachräumen. Die ungarischen Artikel wurden übersetzt, um sie deutschsprachig zugänglich zu machen. Englischsprachige wurden beibehalten. Diese thematische und regionale Ausrichtung war für das Herausgeberteam auch die Leitlinie, die Einladungen zur Mitwirkung an dieser Festschrift auszusprechen.

\section{Stadtgeographie der Nachbarstädte Wien und Budapest}

Den Anfang macht Tamás T. Sikos, der mit Doris WastlWalter bereits im erwähnten Grenzraumprojekt an der österreichisch-ungarischen Grenze zusammengearbeitet 
hat, und mit ihr auch das Interesse an stadtgeographischen Fragen teilt. In seinem Beitrag widmet sich Sikos der Transformation der Stadt Wien, wo Doris WastlWalter ihr Geographiestudium abgeschlossen hat, und dem Erscheinen der Einkaufszentren. Letztere gehören zur Landschaft moderner Metropolen und gestalten das Stadtbild ebenso wie das alltägliche Leben der Bewohner der Stadt und im Umland. Sikos liefert nicht nur ein umfangreiches, detailliertes Bild zur Geschichte einzelner Wiener Shopping Center, er vergleicht diese nach Angebot, Umsatz, Besucherzahl usw., sondern fragt auch danach, wie sich die modernen Einkaufszentren in ihre Umgebung, in das architektonische Gewebe der traditionsbewussten Stadt Wien eingefügt haben. Einige Einkaufszentren spielen auch eine Rolle über die Staatsgrenze hinweg, insofern sie Kunden aus den näheren Regionen Ungarns und der Slowakei anziehen.

Einen vielmehr stadthistorischen Fokus hat der Aufsatz von Pál Beluszky, der sich mit Budapest, der Hauptstadt Ungarns befasst. Beluszky kennt Doris Wastl-Walter ebenfalls aus dem Projekt an der österreich-ungarischen Grenze, setzte sich in seiner Laufbahn aber auch gerne mit Fragen der historischen Geographie auseinander. In seinem Beitrag „Flaggschiff oder Wasserkopf? Budapests Bedeutung und Position in Ungarn" stellt er die Frage, welche Fakten, Tendenzen, Strukturen im Hintergrund der historisch tief verwurzelten und immer wieder heftigen politischen und fachlichen Debatten für und gegen die ungarische Hauptstadt Budapest stehen. Die Geschichte Budapests zeigt laut dem Autor, dass in der Entwicklung der Hauptstadt einerseits die inneren Triebkräfte, vor allem die günstige geographische Lage der Stadt, andererseits die äußeren wirtschaftlichen, politischen Verhältnissen, die Maßnahmen der jeweiligen Machtzentren oft gleichzeitig und eine voneinander untrennbare Rolle gespielt haben. Die (fach)politische Beurteilung der Position, Rolle, oder des „Übergewichts“ Budapests hänge unter anderem davon $a b$, ob die inneren und äußeren Faktoren, Strukturen und der politische Wille der verschiedenen Akteure in Harmonie miteinander stehen.

\section{Multiethnische Nachbarschaften im Karpaten- becken}

István Berényi, der Doris Wastl-Walter aus demselben Projekt kennt wie Tamás T. Sikos und Pál Beluszky, dehnt seine Analyse auf das Gebiet des gesamten Karpatenbeckens aus, jenen Raum, den Doris Wastl-Walter seit mehreren Jahrzehnten intensiv untersucht. Er gibt in seinem Artikel eine historische Beschreibung der Veränderungen der Raumnutzung im Karpatenbecken. In der Analyse werden die wirtschaftlichen Aktivitäten der Menschen, die demographischen Prozesse und Strukturen, die politischen und Machtverhältnisse in ihrer Dynamik und Komplexität als die Faktoren berücksichtigt, welche die Raumnutzung im Karpatenbecken entscheidend geprägt haben. Dabei widmet der Autor seine Aufmerksamkeit auch den Peripherien, vor allem der Grenzregionen, die eine besondere Art der Raumnutzung aufweisen.

2010 bis 2013 war Doris Wastl-Walter Projektleiterin des internationalen Forschungsprojekts TransMig - Integrating (Trans-)national Migrants in Transition States - des Schweizerischen Nationalfonds, in dessen Rahmen Kolleginnen und Kollegen aus Serbien, Ungarn und aus der Schweiz zusammenarbeiteten. Es war dem Herausgeberteam wichtig, dass einige Ergebnisse aus diesem Projekt auch einem deutschsprachigen Publikum zugänglich gemacht werden. Mehrere der Autorinnen und Autoren haben bereits in früheren grenzüberschreitenden Projekten gemeinsam mit der Jubilarin geforscht und publiziert.

Irén Gábrity Molnár, Károly Kocsis, Zoltán Takács und Patrik Tátrai geben einen komplexen historischen Überblick zur Binnen- und internationalen Migration, welche die heute serbische Provinz Vojvodina betrafen. Das Gebiet der Vojvodina war über die Jahrhunderte stets von unterschiedlichen Migrationsprozessen geprägt. Die Migration wurde einerseits durch Kriege oder Machtund Regimewechsel erzwungen, wie zum Beispiel zur Zeit der Jugoslawienkriege in den 1990er-Jahren, in deren Folge Hunderttausende flüchteten. Anderseits folgte die Migration in friedlichen Zeiten dem Modell der klassischen wirtschaftlichen Migration und führte in der Regel von Osten nach Westen und von Süden nach Norden, von den weniger entwickelten in die entwickelten 
Länder oder Regionen. Unabhängig von den Ursachen der Migration, von den Motiven der einzelnen Migrantinnen und Migranten, spielte Ethnizität als kulturelles und symbolisches Kapital in den Migrationswellen, die die Vojvodina betrafen, stets eine wichtige Rolle.

Ágnes Erőss und Monika Mária Váradi untersuchen in ihrem Artikel den Zusammenhang zwischen Migration, Musik und Identität. Anlass dazu boten die sogenannten Jugopartys, die von ungarischen Migranten aus der Vojvodina in Budapest regelmäßig organisiert werden. Diese Partys stellen für die Migrantinnen und Migranten nicht nur einen Platz für gemeinsame Unterhaltung dar, sondern auch eine Gelegenheit, sich durch die Musik und die Stimmung an ihre Jugend und gleichzeitig an die endgültig verlorene Welt Jugoslawiens zu erinnern. Mit der Jugoparty ist nicht nur eine Art apolitische Nostalgie verbunden, diese Feste dienen auch dazu, dass die ungarischen Migranten aus der Vojvodina die „südliche“ Seite ihrer vojvodinischen Identität erleben und zeigen können und sich damit von den in Ungarn lebenden „einheimischen" Ungarn unterscheiden.

Sascha Finger, Doktorand in der Forschungsgruppe von Doris Wastl-Walter, widmet sich in seinem Beitrag jenen Roma-Frauen (Romnija) aus dem Nordosten Ungarns, die seit 2008 in westeuropäischen Städten auf dem StraBenstrich arbeiten. Diese haben in den Jahren davor mit ihren Körpern Prostitutionsorte in Ungarn konstituiert, bis schließlich die Prostitutionsverordnung von staatlicher Seite neu interpretiert wurde. Heute verändern sie Räume in Städten wie Amsterdam und Zürich. Mit ihrer Entscheidung, Sex an legalen Orten in Westeuropa anzubieten, versuchen sie ihre Familien finanziell zu unterstützen. So wurden sie von einer durch ein patriarchalisches System geprägten Romni zur transnationalen Mutter, Arbeitsmigrantin, Ausländerin und Sexarbeiterin. Diese neue Wirklichkeit führte nicht nur zu räumlichen Veränderungen an den verschiedensten Orten in Europa, sondern auch zu einem Wandel mit ethnischen, sozialen und geschlechterspezifischen Dimensionen.

\section{Grenzen überschreiten}

Ksenija Vidmar-Horvat diskutiert in ihrem Beitrag konzeptionelle Fragen der Migration aus Sicht der Border
Theory. Sie weist darauf hin, dass aufgrund von Globalisierungsprozessen moderne Konzepte von nationaler Territorialität und kulturellen Grenzen aufgebrochen werden, und dass die Ideen von Migration und Verwurzelung, Mobilität und Verharrung, Fluidität und Dauerhaftigkeit ihre Stellung als Konzepte, welche die Migrationsdebatten bestimmen, verloren haben. Des Weiteren diskutiert sie die Frage, wie in modernen Nationalstaaten, die sich von homogenen zu ,verflüssigten“ Gesellschaften entwickelt haben, mit Diskursen zur Immigration, oder Kategorien wie „Fremde“ und „Ausländer“ umgegangen wird. Letztlich fragt sie, wie man in einer Zeit, in der Grenzen selber Objekte in Bewegung sind (sei es als extraterritoriale Kontrollpunkte oder als Instrumente sozialer Segregation in einem bestimmten Gebiet), zum Migranten wird. Ist die Staatsbürgerschaft weiterhin das probate Mittel, die Beziehung zum Staat zu beschreiben oder sind andere Faktoren wie wirtschaftliches, soziales oder kulturelles Kapital wichtiger bei der Definition von Zugehörigkeit und Identität?

Der Artikel von Béla Filep befasst sich mit Sprache als Mittel der „Grenzüberschreitung” in multikulturellen Regionen der Slowakei und Serbiens. Filep zeigt auf, welche Rolle Sprache in den interethnischen Beziehungen in Ostmitteleuropa spielt, welche Hürden dabei existieren oder aufgebaut werden, gleichzeitig aber auch, wie diese abgebaut werden können. Dazu diskutiert der Autor einige Beispiele aus der Region, welche auf den Brücken-bauenden Aspekt von Sprache abzielen. Es werden dabei Möglichkeiten aufgezeigt, wie Grenzen mithilfe von Sprache gerade in einem politisch heiklen Umfeld überwunden werden können.

Rossen Koroutchev, ehemaliger Bundesstipendiat der Schweizerischen Eidgenossenschaft bei Doris WastlWalter, untersucht in seinem Beitrag die sozioökonomische Situation im bulgarisch-serbischen Grenzraum und zeigt auf, welche Rolle dabei grenzüberschreitende Prozesse und Aktivitäten sowohl in der Vergangenheit gespielt haben als auch in der Gegenwart einnehmen. Er weist dabei immer auch auf den Einfluss der geopolitischen Veränderungen und politischen Prozesse hin und streicht für die Gegenwart beider Länder die Wichtigkeit der EU heraus.

Vera Pavlakovich-Kochi greift den Titel eines gemein- 
samen Herausgeberinnenwerks mit Doris Wastl-Walter auf, das 2003 unter dem Namen „Challenged Borderlands" die Überwindung von politischen und kulturellen Grenzen in Osteuropa und in Nordamerika diskutierte. In ihrem Artikel mit dem Zusatz „,Then and Now“ blickt Pavlakovich-Kochi zurück auf Jahre der Annäherung an der US-mexikanischen Grenze. Gleichzeitig zeigt sie, dass durch die Politik nach 9/11, die wirtschaftliche Rezession und ungelöste Immigrationsfragen die grenzüberschreitenden Prozesse gestört worden sind. Am Beispiel der (grenzüberschreitenden) Arizona-Sonora Region analysiert die Autorin den Dekonstruktionsprozess in dieser Region und zeigt, wie ein ursprünglich neoliberales Wirtschaftsmodell zuerst in der Lage war, den Grenzraum neu zu definieren und eine Vielzahl von Akteuren anzuziehen, heute aber nicht in der Lage ist, mit den zunehmenden Prozessen der Neukonstruktion von Grenzen und der wachsenden Diskrepanz zwischen grenzüberschreitender Kooperation auf bundesstaatlicher Ebene und Politiken auf nationaler Ebene zurechtzukommen.

Jeannette Regan schließlich greift noch einmal die Frage der Sprache auf, allerdings im akademischen Kontext und vom Blickwinkel des Lehrens und Lernens. Auf Basis ihrer eigenen Erfahrung beschreibt Regan, wie sie das Prinzip von Anam Cara, Gälisch für Seelenfreund, beim Unterrichten von wissenschaftlichem Englisch für Nichtmuttersprachler angewandt hat. Es geht dabei in erster Linie um das Überschreiten von Grenzen zwischen unterschiedlichen Diskursen und um das Verfolgen von Strategien, die beim Lehr- und Lernprozess wichtig sind. In vier Handouts veranschaulicht die Autorin die Formulierung dieser Ziele.

Dank der uneingeschränkten Wertschätzung all der namhaften Wissenschaftlerinnen und Wissenschaftler für Doris Wastl-Walter konnte diese aktuell in der akademischen Welt ein wenig ungewöhnliche Publikation verwirklicht werden und zwar sowohl in einer Printversion als auch durch die Unterstützung des Instituts für Geographie und Regionalforschung der Universität Klagenfurt, an dem sie selbst 17 Jahre tätig war, als OnlineAusgabe der Klagenfurter Geographischen Schriften.

Der besondere Dank der Herausgeber gilt den Autorinnen und Autoren sowie den Übersetzern und Assistentinnen, insbesondere Dina Spörri für das sorgfältige Layout der gedruckten sowie der Online-Version.

Aber Dank von uns langjährigen Wegbegleiterinnen und Wegbegleitern ist vor allem Doris selbst auszusprechen. Dank beispielweise für vieles, das gelernt und von Ihr aus persönlichem Vorbild übernommen werden konnte, für Anregungen und für ein tiefgehendes Misstrauen gegen engstirniges Denken. Für Freundschaft und tiefe Kollegialität. Offenheit. Immer spontane Ermutigung, Aufgaben und erkannte Fragestellungen anzugehen. Großzügigkeit.

\section{For intellectual generosity! And borderless encoura- gement.}

\section{AutorInnen}

Dr. Béla Filep ist wissenschaftlicher Mitarbeiter in der Gruppe für Kulturgeographie am Geographischen Institut der Universität Bern.

Dr. Monika M. Váradi ist wissenschaftliche Mitarbeiterin am Institut für Regionalforschung der Ungarischen Akademie der Wissenschaften in Budapest.

Martin Walter ist Print- und TV-Journalist mit den Arbeitsbereichen Sport, Innenpolitik, gewerbliche Wirtschaft und Österreichs Landwirtschaft. Zudem war er einige Jahre im diplomatischen Dienst tätig.

Mag. Dr. rer. nat. Rudolf Wastl ist Lektor am Institut für Geographie und Regionalforschung der Alpen-Adria Universität Klagenfurt in den Bereichen Humangeographie und Kartographie. Hauptberuflich ist Rudolf Wastl Leiter des Milgeo Teams beim Militärkommando Kärnten und vorwiegend für Fragen der militärischen Raumordnung inklusive Umweltschutz zuständig. Seit 2008 ist er Mitglied im Board of Directors der Military Specialty Group der Association of American Geographers. 